\title{
Diffusion NMR Characterization of Catalytic Silica Supports: A Tortuous Path
}

\author{
Taylor J. Rottreau, ${ }^{\dagger}$ Christopher M. A. Parlett, ${ }^{\ddagger}$ Adam. F. Lee, ${ }^{\ddagger}$, and Rob Evans $^{* \dagger \dagger}$
}

${ }^{\dagger}$ Aston Institute of Materials Research, School of Engineering and Applied Science, and ${ }^{\ddagger}$ European Bioenergy Research Institute, Aston University, Birmingham B4 7ET, United Kingdom

${ }^{\S}$ Department of Chemistry, University of York, York YO10 5DD, United Kingdom

Supporting Information

ABSTRACT: Mesoporous silicas have found widespread application within the field of heterogeneous catalysis. Acid functionalization of such materials, through one-pot or postsynthetic grafting of sulfonic acid groups, imparts activity for fatty acid esterification, with the studious choice of pore geometry facilitating significant rate enhancements. Diffusion NMR has been utilized for the first time to characterize the structure of mesoporous silicas through the transport behavior of systematically related carboxylic acids confined within their mesopore networks. A reduced diffusion coefficient is obtained for species constrained within the 3-dimensional interconnected pores of KIT-6 relative to the 2-dimensional noninterconnected pore

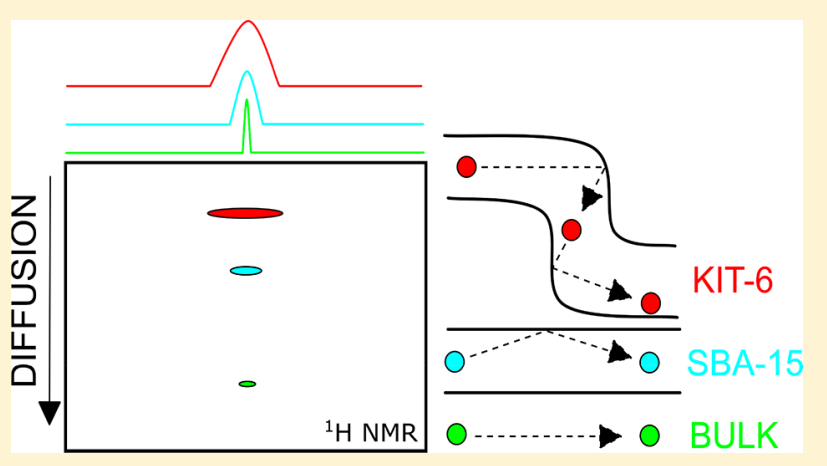
channels of SBA-15. The effective tortuosity of both porous silicas increases with the acid chain length, with the diffusion behavior of long-chain acids dominated by the alkyl chain and silica architecture. Carboxylic acid diffusion within these two pore networks is unlikely to be rate-limiting in catalytic esterification over sulfonic acid silica analogues. Physicochemical insights from diffusion NMR will aid the future design of optimal silica architectures for catalytic applications.

\section{INTRODUCTION}

Porous materials, such as silica, have found widespread use in catalysis due to their variable framework compositions, uniform pore sizes, high thermal stability, and high surface-to-volume ratios. ${ }^{1,2}$ Porous silica supports, including SBA-15 and KIT-6 (Figure 1), have shown potential for the production of biodiesel, ${ }^{3,4}$ with sulfonic acid functionalization resulting in materials that can perform both the transesterification of triglycerides and the esterification of free fatty acid impurities, the latter hindering production of biodiesel over more active base catalytic materials due to poisoning. ${ }^{5,6}$ The effectiveness of these supports toward esterification is highly dependent on the pore structure, with Pirez et $\mathrm{al}^{4}$ showing that reaction rates over sulfonic acid analogues possessing similar size mesopores are enhanced for the 3-dimensional Ia3d cubic architecture of KIT-6 relative to the 2-dimensional $p 6 \mathrm{~mm}$ hexagonal structure of SBA-15. ${ }^{4}$ This rate enhancement is attributed to the different silica architectures and the corresponding effect on the diffusion of reactants and products throughout the support since the two sulfonic acid silicas possessed similar active site densities and operated through an identical catalytic reaction mechanism.

Nuclear magnetic resonance (NMR) methods have been developed to measure the diffusion of small molecules through solution. These can be applied to study molecules in porous materials, using the changes in the properties of a fluid in contact with the surface of the solid to elucidate information about the solid structure. Diffusion NMR, a pulsed field gradient (PFG) technique, ${ }^{7}$ noninvasively measures the displacement of a particular fluid even in constrained environments, such as the porous network of mesoporous silica. The diffusion coefficient of a fluid characterizes its random Brownian motion and gives information about the size of the molecules involved and their local environment. ${ }^{8}$ Such measurements have been established as an effective method of probing the pore geometry and structure, in particular in mesoporous materials where the average pore diameter is between 2 and $50 \mathrm{~nm} .{ }^{9,10}$ The relationship between the bulk diffusion coefficient and the diffusion coefficient of the confined fluid, $D / D_{0}$, yields information on the structural properties of the porous network.

Recent work by Mantle et al. ${ }^{11}$ has studied the diffusion behavior of a series of alcohols in the pores of supported gold catalysts. While most species were hindered by being placed in a porous network, butanediol isomers exhibited an enhancement to their diffusion coefficients in the bulk liquid. It was suggested that this behavior may be down to a disruption of the hydrogen-bonding network by the porous solid. This theory was investigated further by D'Agostino et al., ${ }^{12}$ who studied the

Received: March 28, 2017

Revised: July 8, 2017

Published: July 10, 2017 
(a)

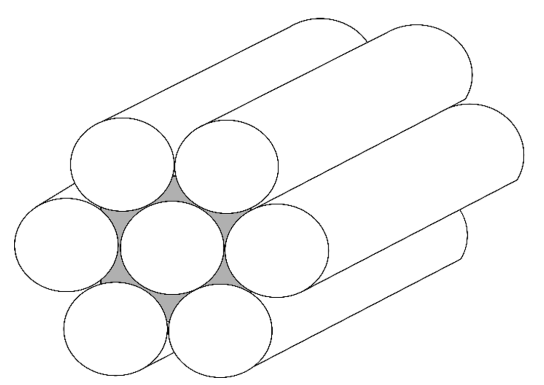

(b)

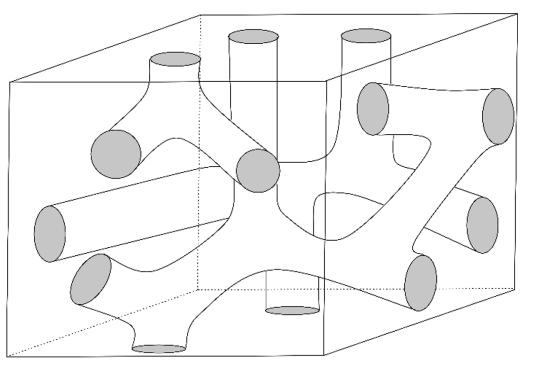

Figure 1. Schematic representation of the (a) parallel cylindrical isolated pores in SBA-15 and (b) cylindrical interconnected pores in KIT-6.

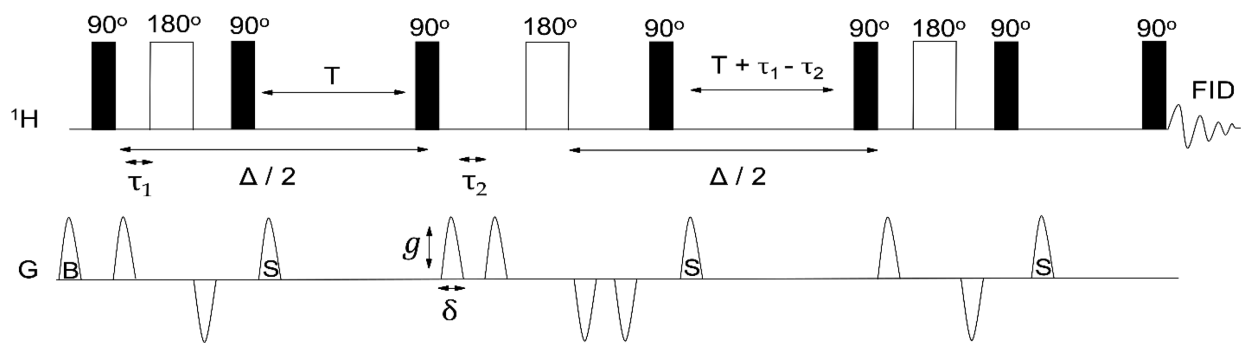

Figure 2. DSTEBPGP_3S pulse sequence for measuring convection-compensated diffusion data.

diffusion of a series of liquids inside different porous environments. An interaction parameter was introduced, $\xi$, the ratio of the diffusion coefficient of the bulk liquid to the diffusion coefficient of the confined liquid. ${ }^{13}$ This ratio is often reported as the tortuosity, $\tau$, of the system. ${ }^{14-16}$ The tortuosity of a material is an inherent property of its structure that defines how twisted and curved the pore walls are. ${ }^{17}$ In this case, where different tortuosities are produced for different chemical species in the pores, $\xi$ rather reflects an effective tortuosity due to the additional interactions of the fluid with the surface of the pore wall. A true value for the tortuosity can be obtained using a noninteracting fluid such as a straight-chained alkane.

In this work, NMR diffusometry was used to characterize catalytic silica supports by studying the in-pore diffusion of reactants and products pertinent to the esterification of fatty acids with methanol within two different silicas. Diffusion coefficients of a series of straight-chain carboxylic acids, as well as alkanes and esters, within the pores of both SBA-15 and KIT-6 were acquired, enabling determination of the effective and actual tortuosities of the two silica catalytic supports. The effect of the alkyl chain length of the fatty acids on their diffusion behavior was also studied. Analysis of reactant and product diffusion behavior within mesoporous silicas offers insight into the different esterification activities of their sulfonated counterparts reported in the literature.

\section{EXPERIMENTAL SECTION}

2.1. Sample Preparation. SBA-15 and KIT-6 silica powders were prepared by the methods of Stucky ${ }^{18}$ and Ryoo, ${ }^{19}$ respectively. Confirmation of their successful syntheses are shown in Figures S1 and S2 (Supporting Information). SBA-15 exhibited wormlike chains comprising high aspect ratio particles around $61 \times 6.5 \times 6.5 \mu \mathrm{m}$, whereas KIT- 6 exhibited more isotropic particles of around $8.4 \times 8.4 \times 8.4 \mu \mathrm{m}$. Propionic acid, valeric acid, hexanoic acid, octanoic acid, lauric acid, myristic acid, palmitic acid, deuterated water, and deuterated methanol were obtained from Sigma-Aldrich and were not subjected to any further purification techniques.
Methyl hexanoate was prepared following a standard esterification procedure outlined elsewhere; ${ }^{20}$ NMR spectra confirming the successful synthesis are included in Figure S3 (Supporting Information). For the diffusion measurements, a $20 \mathrm{mmol}$ solution of each acid was prepared with deuterated methanol. SBA- 15 and KIT-6 were dried in the oven at $100{ }^{\circ} \mathrm{C}$ for $24 \mathrm{~h}$ and then placed in a desiccator for a further $24 \mathrm{~h}$ prior to being used. The acid solution was then pipetted onto each support, and the silica was allowed to soak for at least $12 \mathrm{~h}$. The excess liquid was then removed by carefully blotting the supports on presoaked filter paper. The samples were transferred to a $5 \mathrm{~mm}$ NMR tube, sealed with Parafilm, and placed on a centrifuge at $1500 \mathrm{rpm}$ for $1.5 \mathrm{~h}$. Centrifuging the sample ensures imbibition of the liquid into the pores of the silica. ${ }^{21}$ When placed into the spectrometer, the samples were allowed at least $20 \mathrm{~min}$ before any acquisitions were made to ensure thermal equilibrium had been reached.

2.2. NMR Experiments. All of the measurements were carried out on a Bruker Avance spectrometer with a frequency of $300 \mathrm{MHz}$ and operating at a magnetic field strength of 7.05 $\mathrm{T}$. For diffusion measurements, a $5 \mathrm{~mm}$ PABBO BB-1H ZGRD probe head was used with a maximum gradient of $3.6 \times$ $10^{-1} \mathrm{~T} \mathrm{~m}^{-1}$. The diffusion coefficients were then determined and processed using the DOSY Toolbox software package. ${ }^{22}$ Errors of approximately $1 \%$ in individual diffusion measurements were obtained from the diffusion-ordered spectroscopy (DOSY) fitting of the Stejskal-Tanner equation. However, the main source of error in diffusion experiments is the reproducibility of data acquisition. Parameters such as spectrometer performance and, more significantly, temperature fluctuation will affect the data. This has resulted in a maximum experimental error of $4.0 \%$ for KIT-6 and 3.3\% for measurements in SBA-15. All NMR measurements were made at 298.15 $\mathrm{K}$ and used the double stimulated echo bipolar pulse pair sequence (DSTEBPGP_3S) to remove any possible effects of convection from the diffusion measurements in bulk solution. ${ }^{23,24}$ The same pulse sequence was then used for all later NMR diffusometry experiments. The diffusion delay time, 

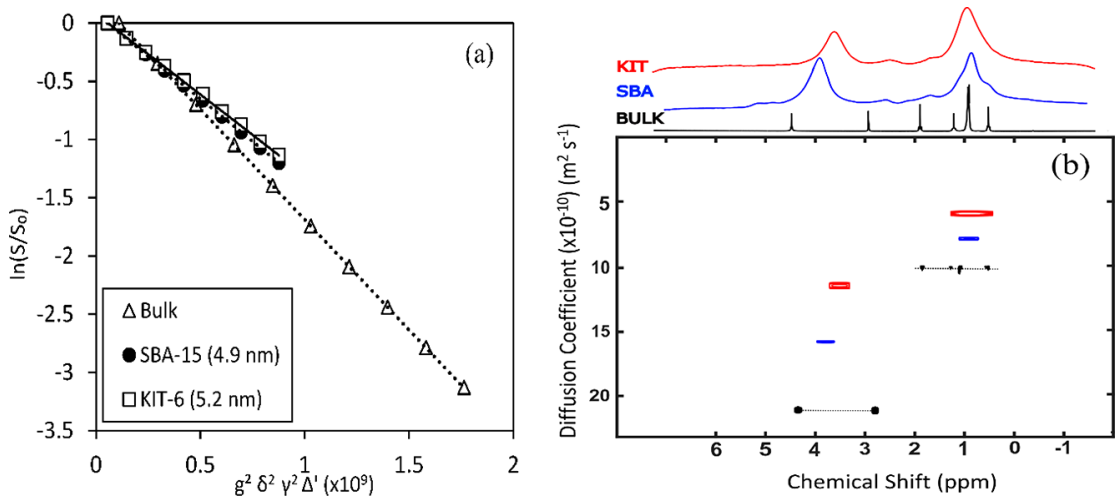

Figure 3. (a) Natural logarithm plot of the signal attenuation versus the gradient strength squared for lauric acid in bulk solution, in SBA-15 (4.9 $\mathrm{nm}$ ), and in KIT-6 (5.2 nm) and (b) DOSY spectra for lauric acid in methanol- $d_{4}$ in bulk solution (black), SBA-15 (blue), and KIT-6 (red).

$\Delta$ (also known as the diffusion drift time ${ }^{25}$ ), is divided into two parts, and the two adjacent sets of bipolar pulse pairs at the center of the sequence have been combined to make sure that only one $180^{\circ}$ pulse is needed to refocus the signal. The sequence contains spoiler gradients, denoted by "S", which are included to remove any unwanted signal in the transverse plane. These are balanced by the gradient pulse, denoted "B". The sequence is illustrated in Figure 2. The signal, $S$, attenuates according to ${ }^{26}$

$$
\frac{S}{S_{0}}=\mathrm{e}^{-D \gamma^{2} \delta^{2} \sigma^{2} g^{2}\left[\Delta+[(2 \kappa-2 \lambda-\delta) / 2]-\left[\left(\tau_{1}+\tau_{2}\right) / 2\right]\right]}
$$

where $S_{0}$ is the signal before a gradient is applied, $D$ is the diffusion coefficient, $\gamma$ is the gyromagnetic ratio of a proton, $\delta$ is the diffusion encoding gradient length $(0.001 \mathrm{~s}), g$ is the diffusion encoding gradient strength, $\tau_{1}$ and $\tau_{2}$ are intergradient delays within the bipolar gradient pulses (both $0.001 \mathrm{~s}$ ), and $\sigma$, $k$, and $\lambda$ are all parameters associated with shaped gradient pulses. $\sigma$ ("gradient area") is an expression of the efficiency of the gradient pulse, and for a half-sine shape, it has a value of 2 / $\pi . k$ and $\lambda$ are further shape parameters used in the StejskalTanner equation and have values of $1 / 2$ and $3 / 8$, respectively, for half-sine gradient pulses. ${ }^{26}$ The delay time, $T$, corresponds to a value of $\Delta=0.3 \mathrm{~s}$ for all the data reported in the main body of the text. Supporting work where the diffusion delays were varied is shown in Figures S19 and S20 (Supporting Information). Ten magnetic field gradient amplitudes from $7.19 \times 10^{-3}$ to $2.87 \times 10^{-2} \mathrm{~T} \mathrm{~m}^{-1}$ were used and incremented in equal steps of gradient squared. For each gradient amplitude, 16 transients of 16384 complex data points were acquired for a total experimental time of $15 \mathrm{~min}$.

\section{RESULTS AND DISCUSSION}

3.1. Tortuosity as a Structural Parameter. The tortuosity of a material can be quantified by measuring the diffusion coefficient of a chemically inactive species confined within a porous material, $D$, and comparing it to that obtained in free solution, $D_{0}$. This ratio of the diffusion coefficient of a species measured in a porous material to that measured when in solution, $D / D_{0}$, is equal to the reciprocal of the tortuosity and allows for the use of diffusion NMR as a means of characterizing the porous material. The tortuosity is commonly used to describe the diffusion behavior of a liquid confined within the pores. ${ }^{9,14,15,27,28}$ Figure 3a shows diffusion NMR data for lauric acid in bulk methanol solution and within two distinct silicas, SBA-15 and KIT-6 with mean mesopore diameters of 4.9 and $5.2 \mathrm{~nm}$, respectively.

Figure $3 b$ is a combined DOSY spectrum of lauric acid in methanol, for bulk methanolic solution, in SBA-15, and in KIT6. When confined within a pore, the acquired diffusion coefficients are lower than for the species measured in bulk solution. The measured diffusion coefficients are in the order bulk > SBA-15 > KIT-6. The further reduction in diffusion coefficient for the acids within KIT-6 compared to SBA-15 suggests that the Brownian motion of the fluids is subjected to increased restrictions. The knowledge that the pores in KIT-6 are interconnected and winding intuitively supports this result.

Diffusion NMR experiments in porous materials can fall into one of two regions: the short diffusion delay time limit and the long diffusion delay time limit. ${ }^{15}$ Taking into account the typical observation times used in an NMR diffusometry experiment, the root-mean-square displacement of a molecule confined within the silica supports exceeds the pore size and falls into the long diffusion delay time limit such that ${ }^{29}$

$$
\Delta \gg \frac{\langle R\rangle^{2}}{6 D}
$$

where $\Delta$ is the diffusion delay time, $R$ is the radius of the pore, and $D$ is the diffusion coefficient. For heptane, one of the species studied here, confined within a pore $4.9 \mathrm{~nm}$ in diameter, $\langle R\rangle^{2} / 6 D$ equates to $1.3 \times 10^{-9} \mathrm{~s}$, which is significantly smaller than diffusion delay times used in the NMR experiments $(0.02-0.30 \mathrm{~s})$. As the diffusion delay time is suitably long enough, the molecules experience many collisions with the walls of the solid, and the reduction in the observed diffusion coefficient will reflect the tortuosity of the solid being studied.

The log attenuation plots produced for the diffusion experiments performed are all straight lines with no deviation, as illustrated in Figure 3a. Nonlinear or curved log attenuation plots can arise for one of a number of reasons: (1) multiple components (e.g., two different diffusing species); (2) the combination of bulk and surface diffusing species (e.g., one species in two different diffusion environments); (3) the rootmean-squared displacement of the diffusing molecule approaching the average pore diameter; (4) instrumental artifacts, such as eddy currents, internal magnetic field gradients, or incomplete phase cycling. ${ }^{11}$ Such linear plots indicate two things: first, that the molecules travel much further than the average pore dimension during the NMR experiment and hence likely interact with the pore walls many times during this period and, second, that, for each measured signal, only one 
species is present, moving with a single diffusion coefficient. Thus, these plots indicate no significant liquid population between particles. All signal attenuation log plots and DOSY spectra for all of the acids measured in this study are provided in Figures S4-S17 (Supporting Information).

Species which possess functional groups, such as carboxylic acids, undergo further interactions with the pore walls. The diffusion measurements now reveal an effective tortuosity, dependent on both the solid structure and surface-liquid interactions. This relationship is shown in the following equation:

$$
\tau_{\text {eff }}=\sigma_{\mathrm{s}} \tau
$$

The term $\sigma_{\mathrm{s}}$ represents the extra interactions between the liquid and the surface and can depend on both the fluid and solid.

The true tortuosity of the solid can be acquired by measuring the diffusion coefficients of noninteracting species such as straight-chain alkanes. The diffusion coefficients of both heptane and cyclohexane were acquired at a series of diffusion delay times, and the tortuosities for the silica supports were obtained for both. Tortuosities obtained for the silica support using the two compounds were identical. Methyl esters are the product of esterification, and hence, the diffusion coefficients of methyl hexanoate were also acquired, both within the porous silica and in solution. The ester exhibited an effective tortuosity within $5 \%$ of the tortuosities obtained using straight-chained alkanes, indicating weaker interactions with the walls of the porous silica than carboxylic acids in line with expectations.

To confirm that this behavior is a characteristic of the pore structures, the tortuosity was measured within the same silica architectures possessing a range of mesopore sizes using cyclohexane and heptane as the noninteracting liquids. The migration of the liquids through the pores should not depend on the pore size of the material if they share the same structures. The results are illustrated in Figure 4. The diffusion coefficients of the alkanes were measured with $\Delta=0.3 \mathrm{~s}$. Further diffusion measurements indicated that, in pores of a smaller diameter, the diffusion was increasingly reduced, indicating an onset of Knudsen diffusion at pore sizes less than approximately 10 times the hydrodynamic diameter of the molecules studied. ${ }^{30,31}$ Above a certain pore size (ca. $4.9 \mathrm{~nm}$ for

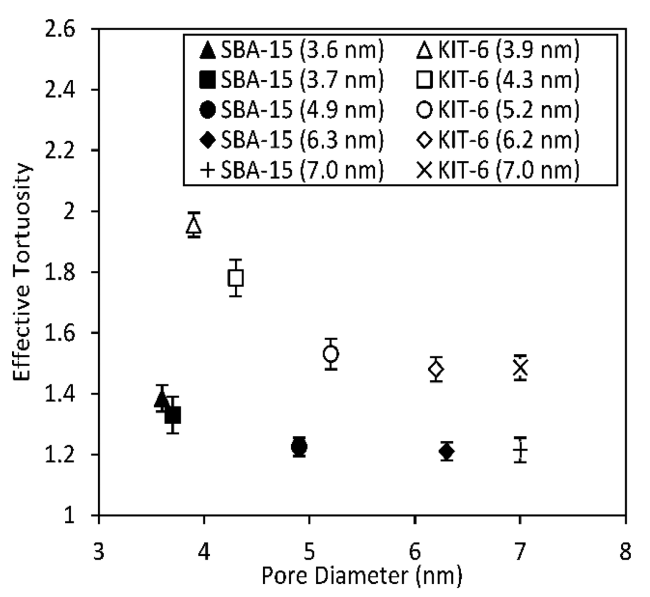

Figure 4. Average values of tortuosity for heptane and cyclohexane confined within the pores of SBA-15 and KIT- 6 . A diffusion delay time of $0.3 \mathrm{~s}$ was used for all measurements to ensure the diffusion was within the long diffusion delay time limit. Values for heptane and cyclohexane were indistinguishable.
SBA-15 and $5.2 \mathrm{~nm}$ for KIT-6; see Figure 4), the effective tortuosity was independent of the pore size for both SBA-15 and KIT-6, with their respective $\tau$ values tending to those in Table 1. As this behavior is observed for both materials and the measurements were all acquired in the long diffusion time limit, it is suggested that it is a property of the supports rather than an artifact of the NMR experiments. These tortuosities are similar to those reported elsewhere for fused $\mathrm{SiO}_{2}\left(\tau \approx 1.6^{12}\right)$.

3.2. Effect of the Chemical Structure on Diffusion. Propanesulfonic acid-functionalized analogues of the two silica supports studied in this work have previously been used in carboxylic acid esterification with methanol, and the resulting kinetic data ${ }^{4}$ indicate that slower diffusion within the twodimensional SBA-15 mesopore channels may result in slower turnover frequencies (TOFs) relative to those of KIT-6 possessing mesopores of a similar average diameter. This hypothesis was therefore investigated through determining the diffusion coefficients of a homologous series of carboxylic acids in the bulk liquid, and when contained within the pores of both SBA-15 and KIT-6 silica supports. The impact of the mesopore diameter of carboxylic acid was also studied for KIT-6.

Figure 5 illustrates the changes in the diffusion coefficient for the homologous series of carboxylic acids in bulk solution, SBA15 (pore size of $4.9 \mathrm{~nm}$ ), and KIT-6 $(5.2 \mathrm{~nm})$. When in the bulk, the acids have no restriction on their motion, and the diffusion coefficients decrease accordingly with increasing chain length-according to the Stokes-Einstein relation. ${ }^{32}$ The same trend of decreasing diffusion coefficients with increasing molecular weight and size is also observed for measurements of shorter chained acids in both porous supports. However, when the chain length of the acids reaches approximately 12 carbon atoms, the observed diffusion coefficients in the silica no longer decrease with increasing chain length and even increase in the case of palmitic acid in KIT-6. When in the pores of the silica, the diffusion coefficient will depend on the bulk diffusion coefficient, the structure of the pores, and any further interactions between the molecule and pore surface, giving rise to the observed differences.

Effective tortuosities, obtained from diffusion data, for all acids studied are shown in Table 2 for a diffusion delay time of $0.3 \mathrm{~s}$. Additional data showing changes in $D / D_{0}$ with reduced diffusion delay times are provided in Figures S18-S20 (Supporting Information), hence confirming that the data presented here were acquired in the long diffusion delay time limit. The carboxylic acids in both silica supports follow the same trend. A small upward trend in diffusion coefficients was observed at shorter values of $\Delta$ for acids in KIT- 6 only. The smallest diffusion delay time used is still significantly larger than that required for the long diffusion time limit, while log attenuation plots of the data remain straight lines; i.e., only one diffusing species is present. This behavior could be due to the presence of restricted diffusion or a boundary within the powder grains. Equation 2 yields an estimate for the KIT-6 particles in the micrometer range consistent with electron microscopy. ${ }^{4}$ Future work is planned to determine the source of this increase in the observed diffusion coefficient.

Figure 6 illustrates that, for short-chain-length acids $\left(C_{n}<6\right)$, the effective tortuosity is lower than the actual tortuosity. This is consistent with the prior work of D'Agostino et al., ${ }^{12}$ where disruption to the hydrogen-bonding network by the solid affects the diffusion of species within it. Smaller molecular weight acids such as propionic acid will experience stronger hydrogen bonding in the bulk solution, and a subsequent 
Table 1. Effective Tortuosity Measured for Noninteracting Species Confined within the Pores of SBA-15 (4.9 nm) and KIT-6 $(5.2 \mathrm{~nm})$

\begin{tabular}{lclc}
\multicolumn{1}{c}{ species } & $D_{0}\left(10^{-10} \mathrm{~m}^{2} \mathrm{~s}^{-1}\right)$ & $\tau(\mathrm{KIT}-6(5.2 \mathrm{~nm}))$ & $\tau(\mathrm{SBA}-15(4.9 \mathrm{~nm}))$ \\
$n$-heptane & $30.10 \pm 0.2$ & $1.48 \pm 0.03(2.1 \%)$ & $1.20 \pm 0.02(1.8 \%)$ \\
cyclohexane & $14.14 \pm 0.17$ & $1.58 \pm 0.05(3.1 \%)$ \\
methyl hexanoate & $13.50 \pm 0.20$ & $1.23 \pm 0.03(2.4 \%)$
\end{tabular}

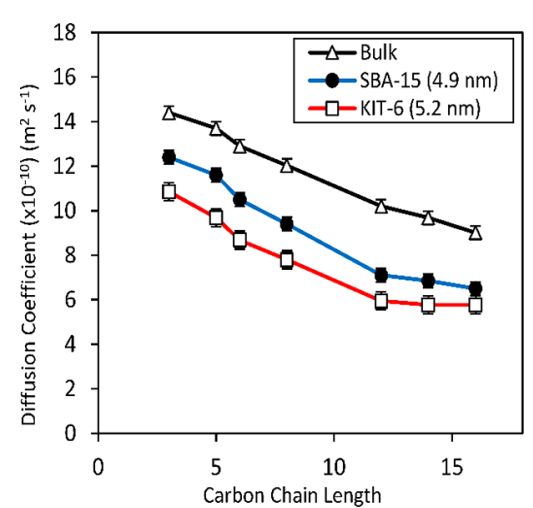

Figure 5. Diffusion coefficients of a homologous series of carboxylic acids acquired in bulk solution, SBA-15 (4.9 nm), and KIT-6 (5.2 nm). A diffusion delay time of $0.3 \mathrm{~s}$ was used for all experiments to ensure that measurements were carried out in the long diffusion delay time limit.

Table 2. Effective Tortuosity Measured for Carboxylic Acids Confined in SBA-15 (4.9 nm) and KIT-6 (5.2 nm)

\begin{tabular}{lcc}
\multicolumn{1}{c}{ acid } & $\tau_{\text {eff }}($ KIT-6) & $\tau_{\text {eff }}$ (SBA-15) \\
propionic acid & $1.33 \pm 0.06(4.6 \%)$ & $1.16 \pm 0.04(3.1 \%)$ \\
valeric acid & $1.41 \pm 0.06(4.0 \%)$ & $1.18 \pm 0.04(3.1 \%)$ \\
hexanoic acid & $1.49 \pm 0.05(3.4 \%)$ & $1.22 \pm 0.04(3.3 \%)$ \\
octanoic acid & $1.54 \pm 0.05(3.5 \%)$ & $1.27 \pm 0.03(2.2 \%)$ \\
lauric acid & $1.72 \pm 0.06(3.2 \%)$ & $1.43 \pm 0.03(2.4 \%)$ \\
myristic acid & $1.68 \pm 0.05(2.7 \%)$ & $1.41 \pm 0.03(2.0 \%)$ \\
palmitic acid & $1.56 \pm 0.05(3.2 \%)$ & $1.39 \pm 0.04(2.5 \%)$ \\
\hline
\end{tabular}

disruption of their networks by the catalytic silica leads to an enhanced diffusion in the porous network.

The effective tortuosity increases with increasing chain length at first, but then, for acids with a chain length of $\mathrm{C}_{12}$ and higher, it starts to fall back toward the tortuosity of the silica itself. This result suggests that, as the alkyl chain increases in size, the effect of the hydrogen-bonding polar acid group decreases. When the size of the acid becomes large enough, the significance of the functionality which defines the strength of the interaction between acid and silica is reduced enough so that the effective tortuosity experienced by the acids eventually tends toward the tortuosity of the system. The geometry of the support is now the determining factor in the diffusional behavior, reducing the significance of the chemistry of the system.

The dependence of tortuosity on the alkane chain length was independent of the pore size (Figures S21 and S22, Supporting Information). The strength of the interaction between silica and acid can be assessed by comparing the effective tortuosity of the acids to the tortuosity of the system obtained from the noninteracting species (see section 3.1). These data are shown in Figure $6 \mathrm{~b}$ and allow the comparison of the behavior of the same acids in the two silica supports studied. The figures analogous to Figure 6 for KIT-6 with larger pore sizes are provided in the Supporting Information (Figures S21 and S22).

Figure 7 compares the enhancement in TOF for carboxylic acid esterification with methanol (Scheme 1) over propanesulfonic acid KIT-6 $(5.2 \mathrm{~nm})$ relative to propanesulfonic acid SBA-15 $(4.9 \mathrm{~nm})$ with the ratio of the effective tortuosities for equivalent unfunctionalized silicas. The ratio of the effective tortuosity of the KIT-6 support to that of the SBA-15 support is a direct, quantitative measure of their structural differences and associated paths taken by reactant/product molecules through them. An increase in the tortuosity implies a more convoluted path through the pore network and hence slower mass transport through a given section of the porous material.

Figure 7 indicates, counterintuitively, an apparent weak correlation between the effective tortuosity of the unfunctionalized mesoporous architectures in this work and observed catalytic performance of their sulfonated analogues, with esterification over the more tortuous sulfonated KIT-6 significantly enhanced relative to that of sulfonated SBA- $15 .^{4}$ However, quantitative analysis of the relative catalytic activity versus effective tortuosity for a range of KIT- 6 mesopore sizes
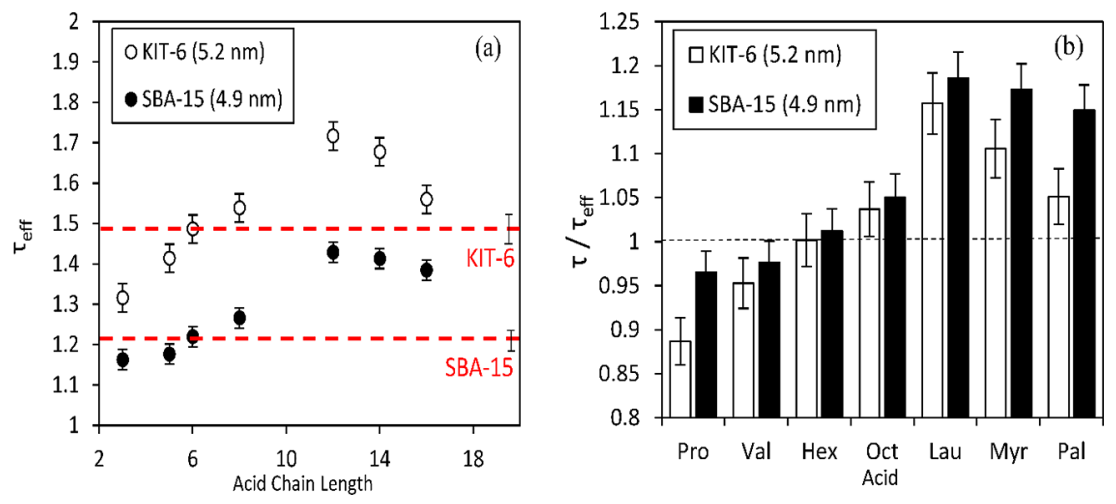

Figure 6. (a) Effective tortuosity for the acids confined within the pores of SBA-15 (4.9 nm) and KIT-6 (5.2 nm) at a diffusion delay time of $0.3 \mathrm{~s}$ and (b) ratio of the structural tortuosity to the effective tortuosity for the homologous series of carboxylic acids in SBA-15 and KIT-6. The dashed line represents the actual tortuosity of the silica, with error bars to the right. 


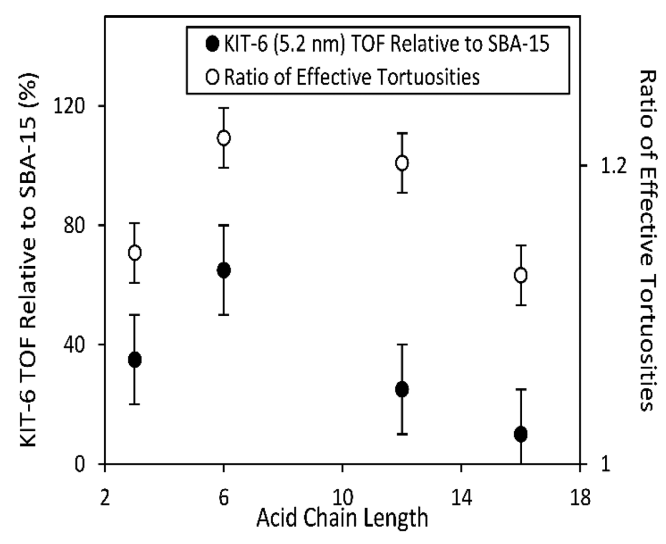

Figure 7. Relative TOF for esterification over propanesulfonic acidfunctionalized KIT-6 $(5.2 \mathrm{~nm})$ versus SBA-15 $(4.9 \mathrm{~nm})$ from ref 4 and ratio of the effective tortuosity of unfunctionalized KIT- 6 and SBA-15 from NMR as a function of the carboxylic acid chain length.

Scheme 1. Catalytic Esterification of Carboxylic Acids with Methanol

$\mathrm{OH} \mathrm{OH}_{\mathrm{n}}+\mathrm{O}_{\mathrm{H}} \longrightarrow-\mathrm{OHN}_{\mathrm{H}}+\mathrm{H}^{-\mathrm{O}_{H}}$

reveals that these two properties are in fact uncorrelated (Figure S23, Supporting Information). This finding is important, since we previously proposed that the superior catalytic activity of sulfonated KIT-6 versus SBA-15 for esterification arose from either enhanced reactant/product diffusion or greater accessibility to the pore network through its higher density of pore openings. ${ }^{4}$ Figures 3 and 5 and Table 1 show that diffusion coefficients are always faster for acid and ester within SBA-15, and hence, the former hypothesis can now be discounted. We therefore attribute the higher esterification activity of sulfonated KIT-6 to its 3-dimensional nature, which affords access to the interior of crystallites via pore entrances uniformly distributed across the entire external surface (in contrast to SBA-15 for which pore entrances preferentially dominate the smaller end faces of wormlike particles). Previous calculations suggest KIT-6 possesses $\sim 6400$ pore entrances $\mu \mathrm{m}^{-3}$ versus only $\sim 290$ pore entrances $\mu \mathrm{m}^{-3}$ for SBA- 15 . ${ }^{4}$ Diffusion NMR is thus shown to be a powerful tool for distinguishing whether in-pore diffusion, or diffusion from the bulk media into a pore network, is likely rate-determining for a heterogeneously catalyzed liquid-phase reaction. This methodology should be broadly applicable to discriminating the impact of the pore architecture on in-pore diffusion of any organic molecule.

\section{CONCLUSIONS}

NMR diffusometry has been used to characterize SBA-15 and KIT-6 silica used as catalytic supports in free fatty acid esterification for biodiesel productions for the first time. The tortuosity of such supports plays a key role in the mass transport of material through the catalyst; however, to the best of our knowledge, this parameter is not widely measured. Differences in the observed rates of reaction occurring in SBA15 and KIT-6 silica are typically rationalized in terms of increased diffusion of the species through the materials; ${ }^{4}$ here we show that in-pore molecular diffusion is unlikely to be ratedetermining in carboxylic acid esterification over sulfonated analogues and provide further insight into the mass transport of reactants and products within a porous support which will aid future catalyst design.

The diffusion coefficients of a homologous series of carboxylic acids are reduced, relative to diffusion in bulk solution, when measured in the pores of the two types of silica supports studied, and this reduction is dependent on both the structure of the host silica, regardless of its average pore diameter, and the structure of the acid. The work shows that the tortuosities of the two silica supports are different. As intuitively expected, the 3-dimensional interconnected system of KIT-6 has a higher tortuosity than the isolated porous system of SBA-15. The effective tortuosities of the two silica supports were measured using a homologous series of carboxylic acids and were found to change with the carbon chain length, implying that an extra interaction was affecting the motion of the acids through the porous materials. The stronger networks of hydrogen bonding associated with smaller acids are broken down by the silica, and slight enhancements to the diffusion are observed. However, longer chained acids exhibit a reduction in apparent diffusion coefficient, due to the tortuous nature of the silica supports. As the alkyl chains of the acids increase further in size, the observed diffusion behavior starts to tend toward that of an alkane, indicating a dependence of the diffusion coefficient on the silica geometry only.

To our knowledge, this represents the first application of NMR diffusometry to characterize the pore networks of catalytically relevant silica supports in regard to their tortuosity. The diffusion behavior of reactants and products related to carboxylic acid esterification provides chemical insight into the likely rate-determining step in fatty acid methyl ester (biodiesel) production. This work demonstrates that widely available NMR methods can be adopted to noninvasively measure the motion of a confined liquid and obtain key information about the structure and chemistry of porous architectures. This methodology should find widespread use in the design and optimization of mesoporous materials with application in liquid-phase chemistry, such as heterogeneous catalysis, separation science, and adsorption.

\section{ASSOCIATED CONTENT}

\section{Supporting Information}

The Supporting Information is available free of charge on the ACS Publications website at DOI: 10.1021/acs.jpcc.7b02929.

Low-angle X-ray diffraction data for both silica supports, ${ }^{1} \mathrm{H}$ NMR spectra for methyl hexanoate, details of the log attenuation plots for all species, DOSY spectra for all acids, effect of changing the diffusion delay time of the measurements, and change in the effective tortuosity for larger pore sizes (PDF)

\section{AUTHOR INFORMATION}

\section{Corresponding Author}

*E-mail: r.evans2@aston.ac.uk. Phone: 0044-(0)121 2045382.

ORCID

Taylor J. Rottreau: 0000-0003-4791-7134

Rob Evans: 0000-0003-1471-201X

Notes

The authors declare no competing financial interest. 


\section{ACKNOWLEDGMENTS}

Financial support for a Ph.D. studentship from the School of Engineering and Applied Science, Aston University, is gratefully acknowledged. A.F.L. and C.M.A.P. were supported by the Engineering and Physical Sciences Research Council (Grant Number EP/N009924/1). We also thank Dr. Paul Topham for his contributions to improving the manuscript.

\section{REFERENCES}

(1) Huirache-Acuna, R.; Nava, R.; Peza-Ledesma, C. L.; LaraRomero, J.; Alonso-Núez, G.; Pawelec, B.; Rivera-Munoz, E. M. SBA15 Mesoporous Silica as Catalytic Support for Hydrodesulfurization Catalysts-Review. Materials 2013, 6, 4139-4167.

(2) Meynen, V.; Cool, P.; Vansant, E. F. Verified Syntheses of Mesoporous Materials. Microporous Mesoporous Mater. 2009, 125, 170-223.

(3) Lee, A. F.; Wilson, K. Sol-Gel Sulfonic Acid Silicas as Catalysts. Handbook of Green Chemistry; Wiley: Chichester, U.K., 2009.

(4) Pirez, C.; Caderon, J. M.; Dacquin, J. P.; Lee, A. F.; Wilson, K. Tunable KIT-6 Mesoporous Sulfonic Acid Catalysts for Fatty Acid Esterification. ACS Catal. 2012, 2, 1607-1614.

(5) BP. BP Energy Outlook 2030 Conference, London, U.K., January 2011.

(6) Montero, J. M.; Gai, P.; Wilson, K.; Lee, A. F. Structure-Sensitive Biodiesel Synthesis over MgO Nanocrystals. Green Chem. 2009, 11, 265-268.

(7) Callaghan, P. T. Principles of Nuclear Magnetic Resonance Microscopy; Clarendon: Oxford, U.K., 1991.

(8) Einstein, A. Investigations on the Theory of Brownian Movement; Dover Publications: New York, 1956.

(9) Sen, P. N. Time-Dependent Diffusion Coefficient as a Probe of Geometry. Concepts Magn. Reson. 2004, 23A, 1-21.

(10) Callaghan, P. T.; Coy, A.; MacGowan, D.; Packer, K. J.; Zelaya, F. O. Diffraction Like Effects in NMR Diffusion Studies of Fluids in Porous Solids. Nature 1991, 351, 467-469.

(11) Mantle, M. D.; Enache, D. I.; Nowicka, E.; Davies, S. P.; Edwards, J. K.; D’Agostino, C.; Mascarenhas, D. P.; Durham, L.; Sankar, M.; Knight, D. W.; et al. Pulsed-Field Gradient NMR Spectroscopic Studies of Alcohols in Supported Gold Catalysts. J. Phys. Chem. C 2011, 115, 1073-1079.

(12) D’Agostino, C.; Mitchell, J.; Gladden, L. F.; Mantle, M. D. Hydrogen Bonding Network Disruption in Mesoporous Catalyst Supports Probed by PFG-NMR Diffusometry and NMR Relaxometry. J. Phys. Chem. C 2012, 116, 8975-8982.

(13) D’Agostino, C.; Kotionova, T.; Mitchell, J.; Miedziak, P. J.; Knight, D. W.; Taylor, S. H.; Hutchings, G. J.; Gladden, L. F.; Mantle, M. D. Solvent Effect and Reactivity Trend in the Aerobic Oxidation of 1,3-Propanediols over Gold Supported on Titania: NMR Diffusion and Relaxation Studies. Chem. - Eur. J. 2013, 19, 11725-11732.

(14) Latour, L. L.; Kleinberg, R. L.; Mitra, P. P.; Sotak, C. H. Pore Size Distributions and Tortuosity in Heterogeneous Porous Media. J. Magn. Reson., Ser. A 1995, 112, 83-91.

(15) Valiullin, R.; Skirda, V. Time Dependent Self-Diffusion Coefficient of Molecules in Porous Media. J. Chem. Phys. 2001, 114, $452-458$.

(16) Gao, X. C.; Dinizda Costa, J. C.; Bhatia, S. K. Understanding the Diffusional Tortuosity of Porous Materials: An Effective Medium Theory Perspective. Chem. Eng. Sci. 2014, 110, 55-71.

(17) Epstein, N. On Totuosity and the Totuosity Factor in Flow and Diffusion Through Porous Media. Chem. Eng. Sci. 1989, 44, 777-779.

(18) Zhao, D.; Feng, J.; Huo, Q.; Melsoh, N.; Fredrickson, G. H.; Chmelka, B. F.; Stucky, G. D. Triblock Copolymer Synthesis of Mesoporous Silica with Periodic 50 to 300 Angstrom Pores. Science 1998, 279, 548-552.

(19) Kleitz, F.; Choi, S. H.; Ryoo, R. Cubic Ia3d Large Mesoporous Silica: Synthesis and Replication to Platinum Nanowires, Carbon Nanorods and Carbon Nanotubes. Chem. Commun. 2003, 2136-2137.
(20) Harwood, L. M.; Moody, C. J.; Percy, J. M. Experimental Organic Chemistry-Standard and Microscale; Wiley: Chichester, U.K., 1999.

(21) Petrov, O.; Furo, I. A Study of Freezing-Melting Hysteresis of Water in Different Porous Materials. Part II: Surfactant-Templated Silicas. Phys. Chem. Chem. Phys. 2011, 13, 16358-16365.

(22) Nilsson, M. The DOSY Toolbox: A New Tool for Processing PFG NMR Diffusion Data. J. Magn. Reson. 2009, 200, 296-302.

(23) Swan, I.; Reid, M.; Howe, P. W.; Connell, M. A.; Nilsson, M.; Moore, M. A.; Morris, G. A. Sample convection in liquid-state NMR: why it is always with us, and what we can do about it. J. Magn. Reson. 2015, 252, 120-9.

(24) Barbosa, T. M.; Rittner, R.; Tormena, C. F.; Morris, G. A.; Nilsson, M. Convection in liquid-state NMR: expect the unexpected. RSC Adv. 2016, 6, 95173-95176.

(25) Hou, J.; Madsen, L. A. New Insights for Accurate Chemically Specific Measurements of Slow Diffusing Molecules. J. Chem. Phys. 2013, 138, 054201.

(26) Sinnaeve, D. The Stejskal-Tanner Equation Generalized for any Gradient Shape-An Overview of Most Pulse Sequences Measuring Free Diffusion. Concepts Magn. Reson., Part A 2012, 40a, 39-65.

(27) Barrie, P. J. Characterization of Porous Media using NMR Methods. Annu. Rep. NMR Spectrosc. 2000, 41, 265-316.

(28) Kärger, J.; Valiullin, R. Diffusion in Porous Media; Wiley: Leipzig, Germany, 2011.

(29) Stallmach, F.; Karger, J. The Potentials of Pulsed Field Gradient NMR for Investigation of Porous Media. Adsorption 1999, 5, 117-133.

(30) Keil, F. J. Diffusion and Reaction in Porous Networks. Catal. Today 1999, 53, 245-258.

(31) Magalhães, F. D.; Laurence, R. L.; Conner, Wm. C. Diffusion of Cyclohexanes and Alkylcylohexanes in Silicalite. J. Phys. Chem. B 1998, 102, 2317-2324.

(32) Einstein, A. The Motion of Elements Suspended in Static Liquids as Claimed in the Molecular Kinetic Theory of Heat. Ann. Phys. 1905, 322, 549-560. 